\title{
Prediction Model of Working Hours of Cooling Turbine of Jet Engine with Back-propagation Neural Network
}

\author{
Ho-Sheng Chen, ${ }^{1}$ Tian-Syung Lan, ${ }^{1,2^{*}}$ and Yu-Ming Lai ${ }^{2}$ \\ ${ }^{1}$ College of Mechatronic Engineering, Guangdong University of Petrochemical Technology, \\ Maoming, Guangdong 525000, China \\ ${ }^{2}$ Department of Information Management, Yu Da University of Science and Technology, \\ Miaoli County 36143, Taiwan
}

(Received July 27 2020; accepted December 3, 2020)

Keywords: environmental control system, back-propagation neural network, prediction model, cooling turbine, airplane inspection

It is critical to maintain jet fighter aircraft so that they are in the best flying condition. Among the various components of aircraft, the environmental control system is crucial as it provides cool air for the cabin and electronic equipment. In this study, we develop a model that predicts the remaining working hours of the critical components of aircraft using a backpropagation neural network (BPNN). First, we adopted the Delphi method with repeated questionnaire surveys to select six key components in the environmental control system, where the cooling turbine was considered the most important component for the prediction model. Then, with the same method, four of the key parameters for the cooling turbine were selected to establish the prediction model. Actual maintenance data from 2009 to 2013 were used for training the neural network to produce the smallest errors between the actual working hours according to inspection results and the predicted working hours. This verification result showed that the correlation and the goodness of fit were above 0.984 and 0.963 , respectively, and the prediction accuracy was $93 \%$. These results indicate that the prediction model with the BPNN can be effectively used to predict the remaining working hours of the cooling turbine. The model can reduce the cost of maintenance and the time lost from the disassembly of components and the halting of flights, as well as unnecessary wear and tear.

\section{Introduction}

In aircraft, the control of the environment is critical to ensure safe flight. Important environmental factors to be controlled precisely in aircraft are the temperature, pressure, ventilation, defogging, pressurization, ventilation and defogging cooling, cockpit sealing, and anti-G suit inflation (for high-speed aircraft). Precise temperature control and ventilation are particularly important as aircraft must maintain a suitable air temperature for the cabin and electronic equipment. In particular, as aircraft have various electronic components in a compact and narrow space, temperature control is extremely important. Maintaining a comfortable cockpit environment is also essential for pilots to fly safely. This is also the case for fighter

*Corresponding author: e-mail: tslan888@yahoo.com.tw https://doi.org/10.18494/SAM.2021.3051 
aircraft (jet fighters). To prevent overheating and ensure the normal operation of electronic equipment, periodic preventive inspection or system tests are mandatory. The current maintenance manual for a jet fighter also recommends periodic testing of the environmental control system by measuring the temperature and pressure. ${ }^{(1-3)}$ Testing also determines the components that need to be replaced. Even with a designated inspection period and manual testing, the reliability is not easily improved as the intervals between inspections and tests are short, which increases the maintenance cost. Although components needing replacement and repair are detected through testing, there might be complicated faults involving multiple components or modules. In this case, it is difficult to find the faulty parts, establish the causes of the faults, and prevent the same faults from occurring in the future. In addition, the complex structure of aircraft and the variable and harsh weather conditions during flights make it difficult to estimate the appropriate period and timing for effective maintenance. As the frequency of inspections and tests needs to be decided carefully with the consideration of many factors such as the service hours, preventing the loss of flight hours, the cost, component failure, and faults of the environmental control system need accurate prediction.

For accurate prediction, machine learning, especially neural networks, can be considered. However, there has been no research on using neural networks for the inspection, maintenance, and prediction of aircraft components and faults. As machine learning uses artificial intelligence (AI) to carry out "reasoning", "knowledge acquisition", and "learning" like a human being, it is assumed to be suitable for the complex systems of aircraft.

An algorithm of machine learning analyzes and obtains rules from data to predict unknown data in the future. Neural networks are one of the approaches in machine learning used to process complicated input data. Algorithms based on neural networks are used in medical science, electronics, information technology, national defense, intelligent manufacturing, smart transportation, and so forth. Therefore, applying neural networks to the development of a prediction model for the maintenance of an environmental control system should improve its overall efficiency and reduce costs and time.

With this background, a back-propagation neural network (BPNN) was chosen to predict the lifetime of key components of an aircraft environmental control system and to develop a maintenance strategy in this study. Applying a BPNN to the maintenance system will allow a practical and accurate predictive model to possibly make up for a lack of information from the manufacturer. ${ }^{(4)}$ The new prediction model with a BPNN can be used as an integral part of the maintenance strategy for the jet fighters of a country.

\section{Background}

\subsection{Environmental control system of jet fighter}

In the environmental control system, bleed air from the engine with a pressure of 120-160 psi is transmitted to the primary part of the heat exchanger through a variable pressure-regulating switch. Here, the air is cooled by the ram air in two stages. The first stage involves the continuous cooling of air from outside according to the movement of the jet fighter, 


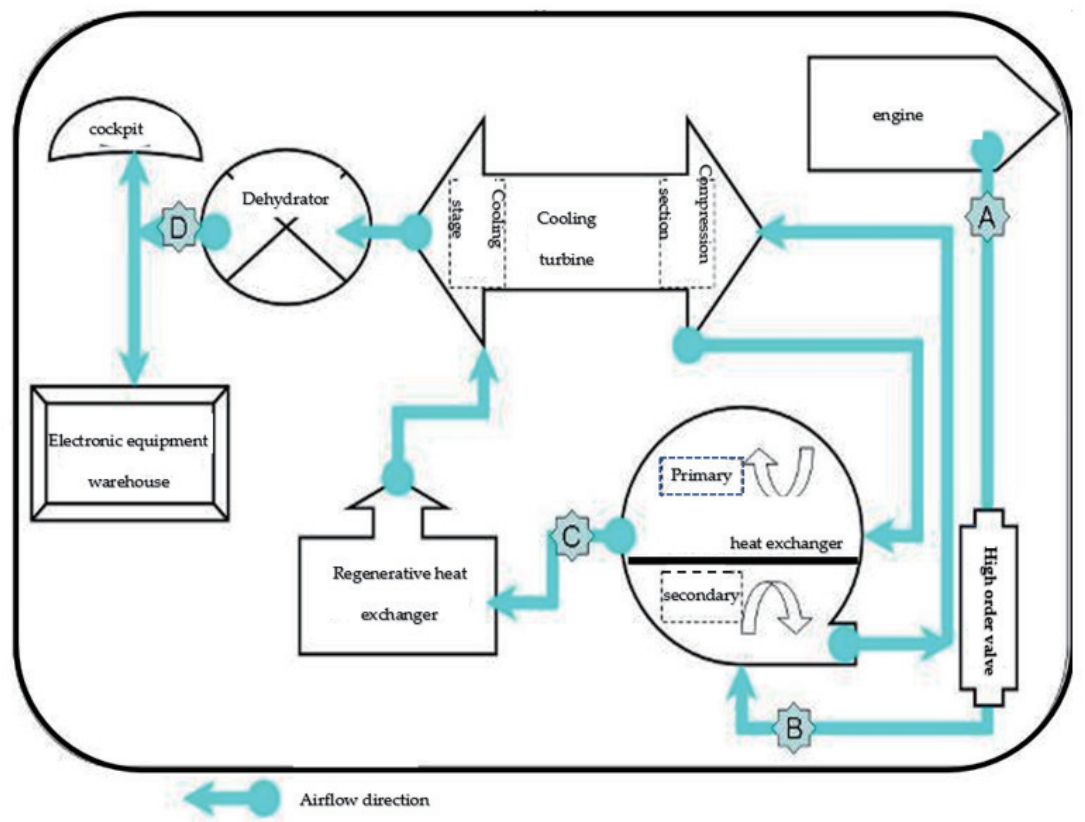

Fig. 1. (Color online) Diagram of environmental control system.

and the second stage involves the cooling of air by an anti-icing water separator and temperature controller and adjuster (Fig. 1).

In the first stage, air is transferred to the compression section of the cooling turbine with a compression ratio of 1.5 to 3.0. At this time, the air temperature increases by about 150 to 260 ${ }^{\circ} \mathrm{F}$ and the pressure changes from 65 to 145 psi. The compressed air from the cooling turbine enters the secondary part of the heat exchanger via the ram air flow. The air is then sent to the regenerative heat exchanger and cooled with water collected by the water separator. Then, it re-enters the cooling section of the cooling turbine. Here, the air temperature is significantly lowered under the turbine's extremely high speed of about 70000 to 80000 revolutions per minute (RPM). The outlet provides cooling air of approximately 32 to $40{ }^{\circ} \mathrm{F}$. The system then imports the outlet air into the water separator, which condenses water using centrifugal force and a specific screen sleeve. When the water droplets are formed, their inertia becomes greater than that of air, and they are expelled out along the inner wall of the separator with the centrifugal rotation. Generally speaking, about $80-85 \%$ of the condensate is removed to complete the cooling stage of the environmental control system. In the system (Fig. 1), the pressure data measured at test point $\mathrm{A}$ is the exhaust air pressure of the engine. The pressure at test point $\mathrm{B}$ transfers to test point $\mathrm{C}$ after compression by the cooling turbine and heat exchange at the primary/secondary heat exchanger, so the air compression ratio of test point $\mathrm{C}$ to test point $\mathrm{B}$ is the ratio of the cooling turbine.

\subsection{Standard test of environmental control system}

H301 environmental control system testers (Howell Instruments) are used for fault detection of the environmental control system of fighter jets (Fig. 2). The tester is connected to the 


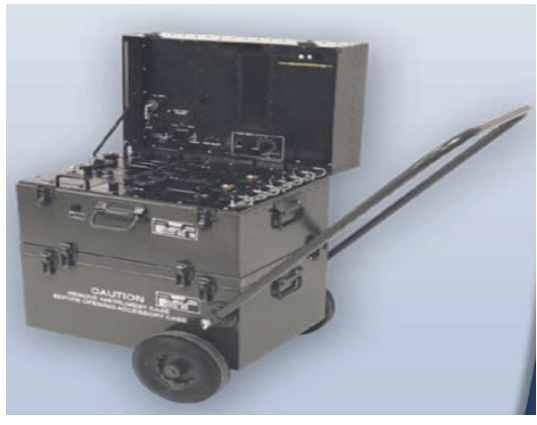

(a)

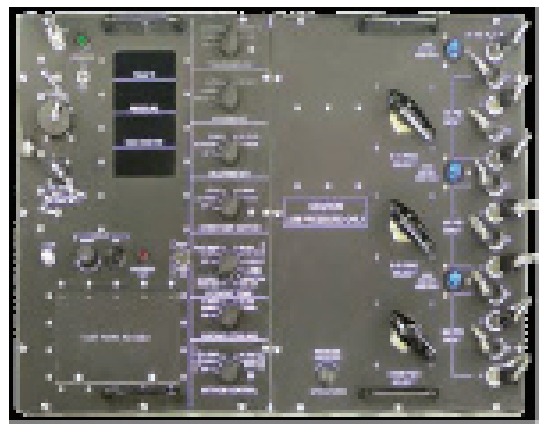

(b)

Fig. 2. (Color online) Environmental control system tester. (a) Photograph of tester and (b) control panel.

power line, high-pressure hose, and temperature sensors at the test points of various parts. The tester measures the temperature and pressure at each test point to determine whether the system provides the correct temperature and pressure of the pipelines in the aircraft cockpit and electronic equipment. The tester also monitors pipeline leakage, the functions and adjustment mechanisms of components, and the refrigeration efficiency of each system.

\subsection{BPNN}

Back-propagation is an algorithm used to train a neural network with supervised learning and has high learning accuracy and a high speed. Its network architecture is based on a multilayer feedforward network with input, hidden, and output layers (Fig. 3).

The basic concept of the BPNN is to minimize the error between the actual and expected outputs, and to train a neural network by using the error so that the actual output of the network is close to the expected output (Fig. 3). The weight of the network moves along the negative gradient of the error function in the weight space, where the weight is updated by Eq. (1). The network is adjusted using the partial derivative of the error function $E$ with respect to the network weight $w$, where $\eta$ is the learning rate used to adjust the weight update range and $n$ is the number of times of training.

$$
\omega(n+1)=w(n)-\eta-\frac{\partial E}{\partial \omega}
$$

The operation of a neural network has two processes: training and simulation. In the training process, the weight value and partial weight of the neural network are determined, while the predicted output value of the network or the accuracy of the network is determined. After entering the neural network from the input layer, the data is passed from the nodes of the input layer to the hidden layer, while the hidden layer and the output node process the incoming information with different weights. All the information of the incoming node is weighted and summed, and then the signal is output from the node through the conversion function. In the training process, the simulation results of each training cycle are produced in the output 


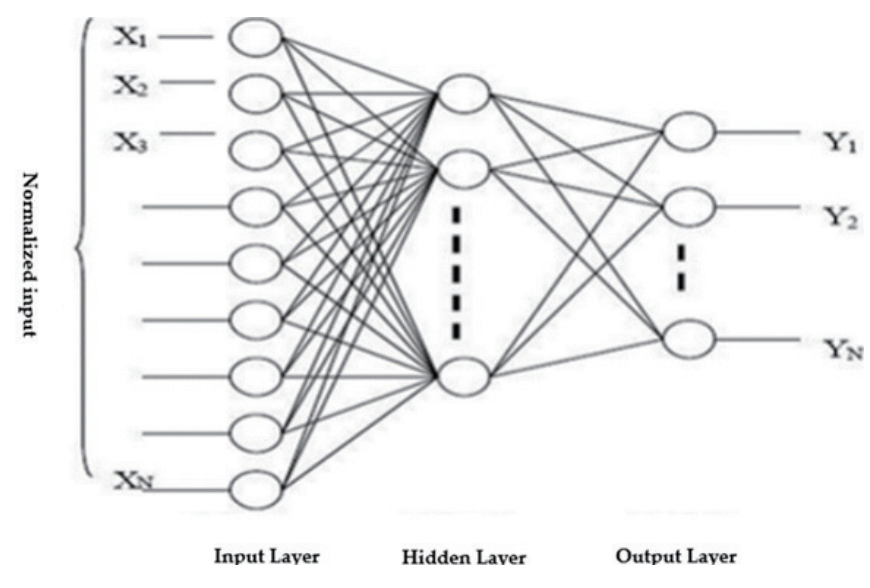

Fig. 3. Structure of BPNN.

layer and compared with a set of known results (training objectives). The errors between the two results are sent back to the neural network to improve its simulation performance. The whole process generates the knowledge of data through the training data via backward error transmission and generates acceptable knowledge via test comparison and correction. The acceptable knowledge is the weight value and partial weight contained in each node.

In general, the BPNN is used to construct a prediction model. The BPNN has a feedforward network architecture as one of the supervised learning networks. The back-propagation uses the input vector and the corresponding target vector to train the neural network. Through the learning and filtering of the network, the network results obtain approximate values from the input variables classified by the transformation function. The BPNN has the characteristic of network generalization. Once the network is trained with representative input values and target values as training datasets, satisfactory output results are obtained for an input that is not in the training dataset. It has the ability of interpolation with the approximation degree of the network. In the BPNN, the unit errors of the output layer are propagated backward layer by layer to the input to obtain the reference error and to adjust the corresponding link weight value. The purpose of this is to minimize the error between the inferred output value and the target output value.

The method of steepest descent is used to minimize the error, i.e., the difference between the predicted value of the network output and the actual target value. The learning quality is generally represented by the sum of the mean squared errors; the smaller the error, the higher the learning quality. In the learning process of the BPNN algorithm, training examples are used to learn and modify repeatedly until it converges. The commonly used transfer functions are logarithmic double bending functions with output values between 0 and 1 , the tangent double bending transfer function with output values between -1 and 1 , and the linear transfer function with output values other than -1 to 1 . Before the prediction of an abnormal trend in a neural network, the effective samples and program tool are selected. After training and learning, the function value is tested with the learning rate, momentum, and training times (i.e., iterative value and epoch) as key factors. 
A too high or too low learning rate adversely affects the convergence of the network. The larger the network weight, the larger the correction of the weighted value. This speeds up the learning and the approximation of the minimum value of the function. However, it also makes the network very unstable. On the other hand, if the learning rate is too low, the convergence speed is low and it is easy to fall into a local minimum value. The learning process adopts the pattern mode. That is, in the learning process, the error is calculated for one epoch, and the weighted value is updated again. This learning cycle is repeated until the final approximation is obtained from 0.1 as a default value, which is adjusted according to the situation of e-learning.

Momentum improves the oscillation of the convergence process and accelerates the convergence of the network. It acts as a low-pass filter, allowing the network to ignore small variations in the error surface and respond to the latest trends. Therefore, the momentum reduces the sensitivity of the network to the local gradient of the error surface to effectively prevent the network from falling into a local minimum.

An epoch refers to one training cycle. When all the data have entered the network for one time of learning, one cycle, i.e., one epoch, is completed. An appropriate number of times of learning gives the network better inductive ability, so that the results accurately predict whether there are no learning samples in the process of testing. Excessive training leads to overlearning, which leads to the inaccurate prediction of new data that have not been learned during the testing process. The problem is solved by determining a reasonable number of times of training. There are two ways to designate the degree of learning. One is to designate the number of times of learning, where the network stops learning after the designated number of times, after which the network stops learning. However, this situation easily leads to the network not converging. The other method is to designate the convergence range of the network and stop learning when the error of the network is less than this value, ensuring that the network converges. If the minimum error is pursued without imposing a condition, the problem of overtraining occurs. This makes the learning error of the network very small but the error of the test result large.

\section{Research Method}

\subsection{Key components of environmental control system}

We adopted the Delphi method to select the key components to be inspected in the environmental control system. This method uses questionnaires to find a solution based on the independent judgments of experts. The Delphi method has been widely accepted and applied to the fields of management and science and technology, such as in the forecasting of technologies and markets. ${ }^{(5,6)}$ Delphi questionnaires are designed to find research topics (the first questionnaire) and ensure their approval (the second or third one) by experts in the relevant field. After the second or third questionnaire is collected, statistical analysis is conducted to obtain the result. In this method, all opinions from experts are reflected in the questionnaire survey, which avoids unnecessary interference.

In this study, questionnaires were sent out to a total of 15 people, on the basis of which we chose the following six key components: high-level engine drain valve, primary/secondary 
heat exchanger, cooling turbine, regenerative heat exchanger, dehydrator, and sensor controller of electronic equipment (Table 1). From the second and third questionnaire surveys, the cooling turbine was selected as the most important component affecting the efficiency of the environmental control system.

\subsection{Likert five-point scale}

To find the key parameters for inspecting the cooling turbine, we accumulated the relevant test data from maintenance manuals for similar jet engines to that in this study (we are not allowed to reveal the name of the engine that was inspected in this study). The questionnaires had a Likert five-point scale. Thus, the experts assigned 1-5 points according to the importance of each parameter (from "least important" to "most important"). The scores given by the experts are shown in Table 2. Parameters with an average score higher than 4 were classified as

Table 1

Results of questionnaire sent to experts.

\begin{tabular}{lccc}
\hline Number & $\begin{array}{c}\text { First questionnaire } \\
\text { Screening key components }\end{array}$ & $\begin{array}{c}\text { Second questionnaire } \\
\text { number of responses }\end{array}$ & $\begin{array}{c}\text { Third questionnaire } \\
\text { number of responses }\end{array}$ \\
\hline 1 & Engine drain valve & 0 & 0 \\
2 & Primary/secondary heat exchanger & 2 & 6 \\
3 & Cooling turbine & 3 & \\
4 & Regenerative heat exchanger & 0 & 0 \\
5 & Dehydrator & 0 & 0 \\
6 & Electronic equipment cabin sensor controller & 1 & \\
\hline
\end{tabular}

Table 2

Results of rating questionnaire sent to experts with Likert five-point scale.

\begin{tabular}{|c|c|c|c|c|c|c|c|c|c|}
\hline \multirow[b]{2}{*}{$\begin{array}{l}\text { Key } \\
\text { factor }\end{array}$} & \multicolumn{9}{|c|}{ Expert rating } \\
\hline & $\begin{array}{l}\text { Air } \\
\text { compression } \\
\text { ratio }\end{array}$ & $\begin{array}{c}\text { Turbine } \\
\text { outlet } \\
\text { temperature }\end{array}$ & $\begin{array}{c}\text { Flight hours } \\
\text { (operation hours) } \\
\text { with installed } \\
\text { turbine }\end{array}$ & $\begin{array}{l}\text { Exhaust air } \\
\text { pressure of } \\
\text { engine }\end{array}$ & $\begin{array}{l}\text { Relative } \\
\text { humidity }\end{array}$ & $\begin{array}{l}\text { Flight } \\
\text { altitude }\end{array}$ & Airspeed & $\begin{array}{l}\text { Engine } \\
\text { speed }\end{array}$ & $\begin{array}{c}\text { Turn on } \\
\text { radar }\end{array}$ \\
\hline 1 & 5 & 4 & 3 & 4 & 5 & 4 & 3 & 3 & 1 \\
\hline 2 & 4 & 4 & 4 & 5 & 4 & 3 & 3 & 3 & 4 \\
\hline 3 & 4 & 5 & 4 & 5 & 5 & 2 & 4 & 3 & 2 \\
\hline 4 & 4 & 5 & 5 & 5 & 3 & 4 & 3 & 4 & 2 \\
\hline 5 & 5 & 4 & 4 & 5 & 4 & 3 & 2 & 1 & 2 \\
\hline 6 & 4 & 5 & 4 & 4 & 2 & 3 & 3 & 2 & 3 \\
\hline 7 & 5 & 5 & 5 & 3 & 1 & 2 & 2 & 2 & 2 \\
\hline 8 & 5 & 4 & 3 & 3 & 1 & 2 & 1 & 2 & 2 \\
\hline 9 & 3 & 4 & 4 & 2 & 1 & 1 & 1 & 2 & 3 \\
\hline 10 & 5 & 2 & 4 & 3 & 1 & 2 & 1 & 2 & 1 \\
\hline 11 & 4 & 4 & 4 & 4 & 1 & 1 & 2 & 1 & 2 \\
\hline 12 & 4 & 4 & 5 & 5 & 1 & 1 & 1 & 1 & 2 \\
\hline 13 & 5 & 4 & 4 & 4 & 2 & 1 & 1 & 1 & 1 \\
\hline Total score & 57 & 54 & 53 & 52 & 31 & 29 & 27 & 27 & 27 \\
\hline Average & 4.38 & 4.15 & 4.08 & 4.00 & 2.38 & 2.23 & 2.08 & 2.08 & 2.08 \\
\hline $\begin{array}{l}\text { Standard } \\
\text { deviation }\end{array}$ & 0.62 & 0.77 & 0.62 & 0.96 & 1.55 & 1.05 & 1.00 & 0.92 & 0.83 \\
\hline
\end{tabular}


key parameters. We found four key parameters: air compression ratio in turbine, turbine outlet temperature, flight hours (operation hours) with installed turbine, and exhaust air pressure of engine. These were the main input parameters of the software in this study.

The four key parameters are described as follows:

(1) Air compression ratio in turbine

The air compression ratio is defined as

$$
\text { Air compression ratio }=\frac{\text { Outlet pressure of cooling turbine }}{\text { Inlet pressure of cooling turbine }} .
$$

According to the manual, the ratio should be greater than 1.5.

(2) Turbine outlet temperature

To evaluate the cooling efficiency of the cooling turbine, the air temperature in the final outlet of the cooling turbine after dehydration is measured (a temperature of between 32 and $40^{\circ} \mathrm{F}$ is specified in the manual).

(3) Flight hours (operation hours) with installed turbine

If a turbine is disassembled for maintenance and then reinstalled on a jet airplane, the flight hours with the installed turbine are then reset to zero and accumulated again.

(4) Temperature and pressure of exhaust air

The temperature and pressure of the air exhausted from the engine are set for the air source in the cooling turbine operation.

\subsection{Prediction model with BPNN}

We used the above four key parameters for a BPNN. In this study, the number of epochs was set from 10000 to 30000 cycles to ensure that the neural network converges. After defining the input and output layers of the prediction model, the flight hours and the data of the cooling turbine were imported into the neural network, and the network parameters were set to establish the prediction model. The main process of the model is as follows:

(1) Setting of input and output layers

To predict the service life of the cooling turbine, the four key factors were set in the input layer, and "flight hours after fault detection" was set as the target value in the output. The prediction model of the service life of the cooling turbine was established by learning and calculation by the neural network.

(2) The number of layers, architecture, and number of neurons in the neural network were determined.

As shown in Table 3, the input layer, hidden layer, and output layer had four, six, and one neuron, respectively. The structure of the neural network parameters is shown in Fig. 4.

(3) Loading fault data of cooling turbine

We selected 57 maintenance datasets of the faults of the cooling turbines of fighter jets in Taiwan in the past four years. Seven of the datasets with less than 30 flight hours were deleted to ensure an effective prediction model. Thirty-four of the 50 remaining datasets $(68 \%)$ were 
Table 3

Data of the prediction model.

\begin{tabular}{|c|c|c|c|c|c|}
\hline \multirow[b]{2}{*}{ Item } & \multicolumn{4}{|c|}{ Input layer } & \multirow{2}{*}{$\begin{array}{c}\text { Output layer } \\
\text { Working hours after } \\
\text { fault detection }(\mathrm{h})\end{array}$} \\
\hline & $\begin{array}{l}\text { Flight hours with } \\
\text { installed turbine (h) }\end{array}$ & $\begin{array}{c}\text { Exhaust air } \\
\text { pressure (psi) }\end{array}$ & $\begin{array}{c}\text { Turbine air } \\
\text { compression ratio }\end{array}$ & $\begin{array}{c}\text { Turbine outlet } \\
\text { temperature }\left({ }^{\circ} \mathrm{F}\right)\end{array}$ & \\
\hline 1 & 260.25 & 137 & 1.60 & 35 & 68.25 \\
\hline 2 & 1762.90 & 136 & 1.53 & 36 & 18.10 \\
\hline 3 & 2013.75 & 137 & 1.55 & 37 & 18.15 \\
\hline 4 & 1010.08 & 137 & 1.65 & 34 & 48.42 \\
\hline 5 & 371.00 & 142 & 1.69 & 36 & 53.85 \\
\hline 6 & 1087.50 & 130 & 1.53 & 38 & 10.75 \\
\hline 7 & 2030.35 & 150 & 1.60 & 32 & 34.90 \\
\hline 8 & 96.30 & 134 & 1.54 & 34 & 44.00 \\
\hline 9 & 442.65 & 139 & 1.80 & 35 & 71.50 \\
\hline 10 & 1626.50 & 141 & 1.87 & 35 & 59.75 \\
\hline 11 & 430.50 & 134 & 1.79 & 38 & 35.80 \\
\hline 12 & 157.90 & 142 & 1.75 & 35 & 23.25 \\
\hline 13 & 270.91 & 145 & 1.59 & 36 & 60.42 \\
\hline 14 & 291.75 & 145 & 1.67 & 35 & 39.58 \\
\hline 15 & 198.92 & 141 & 1.56 & 36 & 46.50 \\
\hline 16 & 1318.58 & 146 & 1.53 & 38 & 7.67 \\
\hline 17 & 346.08 & 142 & 1.69 & 36 & 78.75 \\
\hline 18 & 96.30 & 134 & 1.63 & 36 & 44.00 \\
\hline 19 & 118.30 & 131 & 1.56 & 39 & 22.00 \\
\hline 20 & 442.65 & 139 & 1.67 & 35 & 71.50 \\
\hline 21 & 420.73 & 134 & 1.80 & 35 & 93.42 \\
\hline 22 & 430.50 & 134 & 1.79 & 38 & 35.80 \\
\hline 23 & 1722.66 & 136 & 1.53 & 37 & 18.17 \\
\hline 24 & 153.42 & 147 & 1.77 & 34 & 27.75 \\
\hline 25 & 160.84 & 142 & 1.75 & 36 & 20.33 \\
\hline 26 & 577.50 & 142 & 1.58 & 37 & 18.17 \\
\hline 27 & 995.00 & 145 & 1.53 & 34 & 79.83 \\
\hline 28 & 1025.08 & 136 & 1.59 & 36 & 49.75 \\
\hline 29 & 1626.50 & 141 & 1.87 & 33 & 59.75 \\
\hline 30 & 1010.08 & 137 & 1.65 & 37 & 48.42 \\
\hline 31 & 1366.60 & 142 & 1.57 & 36 & 42.78 \\
\hline 32 & 221.87 & 145 & 1.57 & 36 & 14.33 \\
\hline 33 & 573.49 & 145 & 1.67 & 36 & 92.70 \\
\hline 34 & 1350.73 & 141 & 1.63 & 37 & 28.67 \\
\hline 35 & 65.06 & 146 & 1.59 & 38 & 20.44 \\
\hline 36 & 619.37 & 142 & 1.56 & 38 & 22.40 \\
\hline 37 & 163.54 & 134 & 1.62 & 37 & 46.43 \\
\hline 38 & 327.59 & 131 & 1.88 & 35 & 123.44 \\
\hline 39 & 992.05 & 139 & 1.57 & 33 & 73.10 \\
\hline 40 & 176.17 & 134 & 1.78 & 34 & 158.17 \\
\hline 41 & 468.88 & 134 & 1.62 & 32 & 46.60 \\
\hline 42 & 1268.35 & 136 & 1.70 & 34 & 79.20 \\
\hline 43 & 645.28 & 147 & 1.73 & 35 & 96.15 \\
\hline 44 & 554.29 & 142 & 1.64 & 36 & 79.30 \\
\hline 45 & 718.59 & 151 & 1.86 & 40 & 11.39 \\
\hline 46 & 1163.55 & 145 & 1.59 & 35 & 66.58 \\
\hline 47 & 177.44 & 136 & 1.82 & 34 & 179.17 \\
\hline 48 & 897.61 & 131 & 1.55 & 34 & 46.12 \\
\hline 49 & 369.48 & 139 & 1.79 & 35 & 146.32 \\
\hline 50 & 389.06 & 134 & 1.56 & 35 & 55.36 \\
\hline
\end{tabular}




\section{4-6-1}

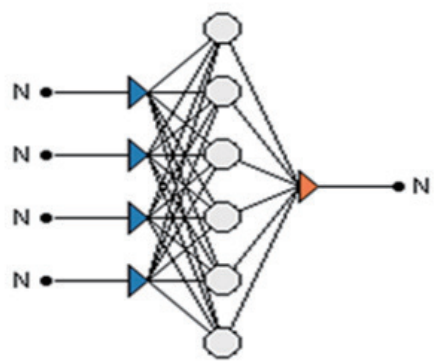

Fig. 4. (Color online) Structure of parameters of neural network.

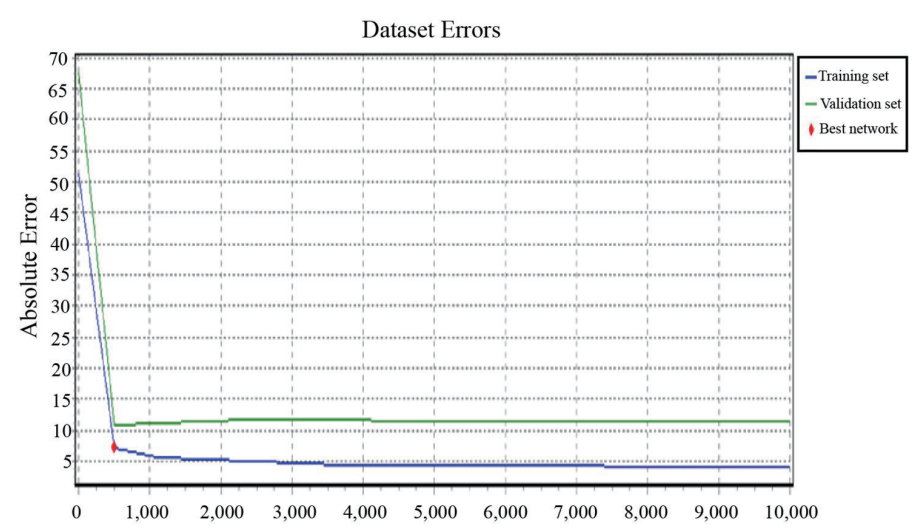

Fig. 5. (Color online) Input and output values with learning rate of 0.1.

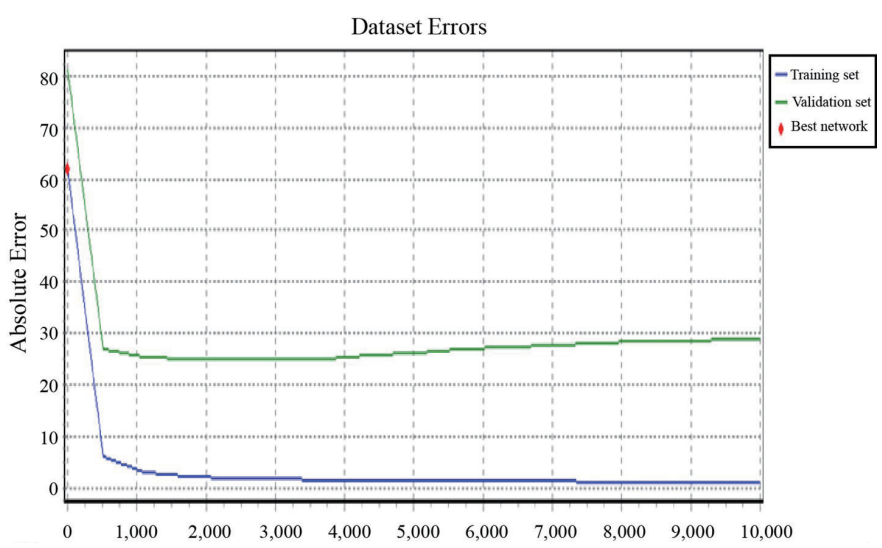

Fig. 6. (Color online) Input and output values with learning rate of 0.15 .

selected as the training data, eight sets $(16 \%)$ were used as validation data, and eight sets $(16 \%)$ were used as test data.

(4) Optimal learning rate

After testing with learning rates from 0 to 100, we found that the error between the input (training set) and output (validation set) values with a learning rate of 0.1 was minimal and converged to the error giving the best learning of the network (Fig. 5). In fact, the error was minimal when the learning rate was 0.15 (Fig. 6), but the error rate did not converge as the error 
between the input (training set) and output (validation set) values was larger than that with the learning rate of 0.15 . Therefore, we selected 0.1 as the best learning rate.

(5) Optimal number of times of training

As shown in Figs. 7 to 9, after training with different numbers of times of learning, we found that convergence started from the 9000th epoch. To ensure the stability of convergence, learning was repeated 20000 and 30000 times. However, convergence was obtained before the 10000th epoch, so we performed learning 10000 times.

\section{Results and Discussion}

With the above process and parameters, we used Alyuda NeuroIntelligence 2.1 to establish an optimal prediction model of high accuracy and analyze parameter sensitivity with the predicted results. The results are given in the following subsections.

\subsection{Training of predictive model}

By repeating the training procedure, validation, and testing 600 times, 20 datasets with relevance and matching ratios close to 1 were selected (Table 4). The datasets had averages of 0.984129 for relevance and 0.963650 for model matching, which were positively correlated. ${ }^{(7)}$ To

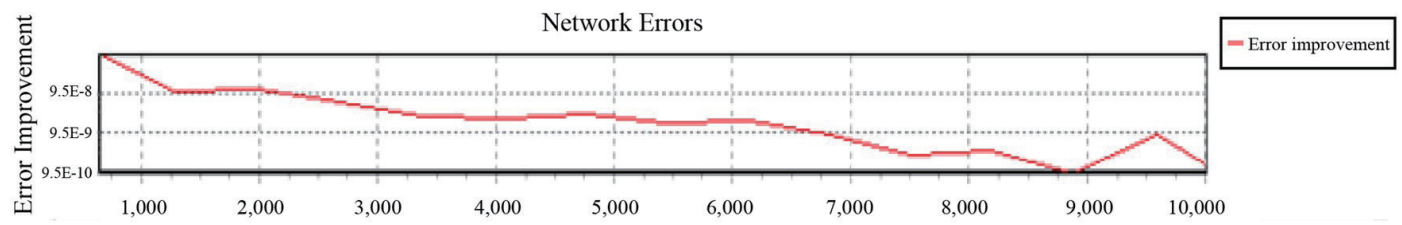

Fig. 7. (Color online) Convergence of error rate for 10000 epochs.

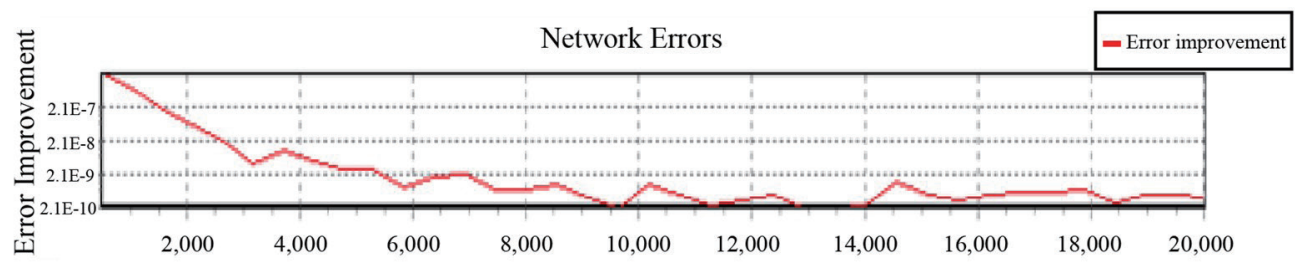

Fig. 8. (Color online) Convergence of error rate for 20000 epochs.

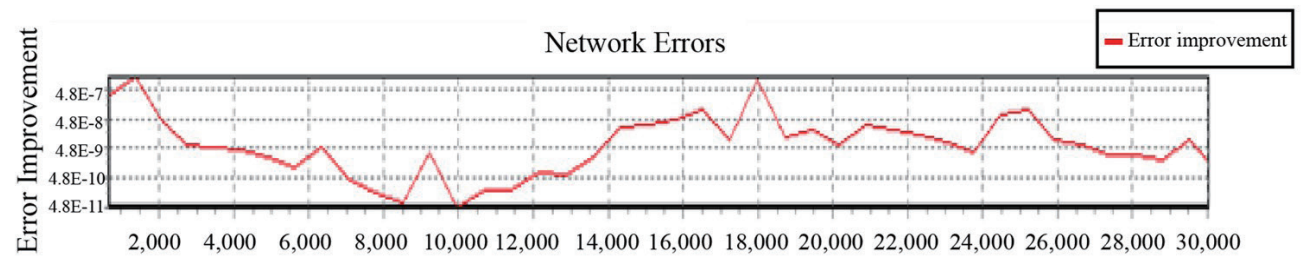

Fig. 9. (Color online) Convergence of error rate for 30000 epochs. 
Table 4

Relevance and model matching of 20 selected datasets.

\begin{tabular}{lcc}
\hline Group & Relevance & Model matching \\
\hline 1 & 0.990664 & 0.979507 \\
2 & 0.990246 & 0.960357 \\
3 & 0.990230 & 0.979544 \\
4 & 0.990122 & 0.979057 \\
5 & 0.988415 & 0.969932 \\
6 & 0.987423 & 0.967494 \\
7 & 0.987246 & 0.960357 \\
8 & 0.986246 & 0.960357 \\
9 & 0.986003 & 0.970782 \\
10 & 0.985037 & 0.967782 \\
11 & 0.984525 & 0.968082 \\
12 & 0.984467 & 0.969386 \\
13 & 0.982619 & 0.961632 \\
14 & 0.981828 & 0.941232 \\
15 & 0.981626 & 0.944207 \\
16 & 0.980778 & 0.970332 \\
17 & 0.979619 & 0.965532 \\
18 & 0.977410 & 0.942043 \\
19 & 0.975201 & 0.962482 \\
20 & 0.972992 & 0.952911 \\
\hline Average & 0.984129 & 0.963650 \\
\hline
\end{tabular}

maximize the prediction accuracy, the optimal prediction model of this study was established with the best correlation and pattern matching as shown in Table 4. Figure 10 shows the difference between the target values and the predicted values for the flight hours of the detected backspace fault. The closer the two curves, the higher the accuracy of the prediction model. Table 5 shows a summary of the prediction by the model in this study. The prediction result showed a goodness of fit of 0.980 , implying that the model closely fit the input data.

\subsection{Life prediction}

After the establishment of the prediction model, 16 datasets of the turbine outlet temperature from January to April 2014 were obtained from the maintenance information of the fighter jet in this study. The predicted value (output value) of flight hours with the installed turbine was obtained after the neural network imported the test as shown in Table 6. After verification and comparison with 16 target values for detecting the backspace failure, mean absolute error (MAE) and mean absolute percentage error (MAPE) were calculated. In this study, the prediction model of the cooling turbine life after inspection had a high accuracy of $90.3 \%$.

Using the prediction model, the average flight hours from 2009 to 2013 were taken as the input data for prediction training. For the prediction of the temperature and pressure of exhaust air, we input $140 \mathrm{psi}$ as the pressure and $700 \mathrm{~h}$ as the flight hours for the installed turbine. In addition, the parameters were prioritized: the turbine air compression ratio was changed first, followed by the turbine outlet temperature, in accordance with the order of importance selected by the experts in the questionnaire. The relevant variation was $1 \%$ of the standard specification. 


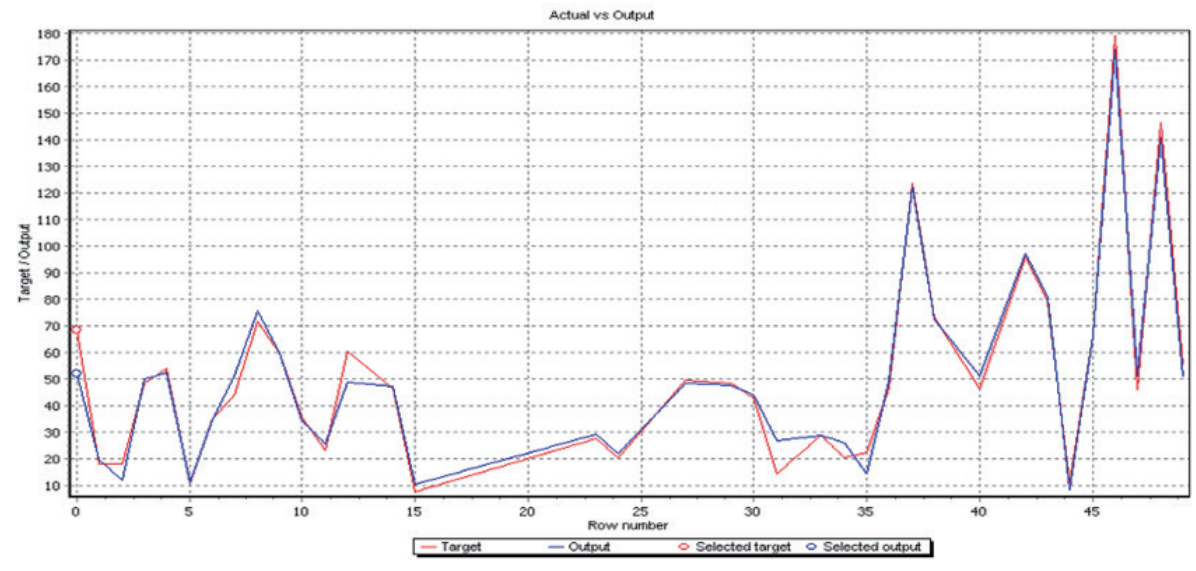

Fig. 10. (Color online) Comparison between target and predicted values.

Table 5

Summary of model prediction.

\begin{tabular}{lcccc}
\hline Statistics & Target value & Output value & Absolute error & Relative error \\
\hline Average & 51.281667 & 51.091372 & 3.51558 & 0.117002 \\
Standard deviation & 36.900563 & 35.68677 & 3.7067 & 0.167242 \\
\hline \multicolumn{5}{c}{ Relevance: 0.990664} \\
\hline
\end{tabular}

Table 6

Life prediction accuracy.

\begin{tabular}{lccccccc}
\hline No. & $\begin{array}{c}\text { Flight hours } \\
\text { with installed } \\
\text { turbine }(\mathrm{h})\end{array}$ & $\begin{array}{c}\text { Exhaust air } \\
\text { pressure } \\
(\mathrm{psi})\end{array}$ & $\begin{array}{c}\text { Turbine air } \\
\text { compression } \\
\text { ratio }\end{array}$ & $\begin{array}{c}\text { Turbine outlet } \\
\text { temperature } \\
\left({ }^{\circ} \mathrm{F}\right)\end{array}$ & $\begin{array}{c}\text { Hours after fault } \\
\text { detection } \\
(\mathrm{h})\end{array}$ & $\begin{array}{c}\text { Predicted } \\
\text { value }\end{array}$ & $\begin{array}{c}\text { MAE } \\
(\%)\end{array}$ \\
\hline 1 & 730.5 & 134 & 1.76 & 35 & 235.80 & 248.11531 & 5.2 \\
2 & 94.8 & 142 & 1.55 & 34 & 127.25 & 129.7765 & 2.0 \\
3 & 470.9 & 145 & 1.59 & 36 & 60.42 & 59.411836 & 1.7 \\
4 & 291.8 & 145 & 1.79 & 33 & 194.58 & 218.08948 & 12.1 \\
5 & 198.9 & 141 & 1.56 & 36 & 246.50 & 216.8794 & 12.0 \\
6 & 78.6 & 146 & 1.53 & 34 & 84.67 & 92.996718 & 9.8 \\
7 & 346.1 & 142 & 1.62 & 36 & 62.75 & 57.189188 & 8.9 \\
8 & 896.3 & 134 & 1.63 & 36 & 44.00 & 49.618939 & 12.8 \\
9 & 918.3 & 131 & 1.56 & 39 & 9.25 & 8.782355 & 5.1 \\
10 & 677.8 & 137 & 1.75 & 36 & 69.80 & 70.534143 & 1.1 \\
11 & 1033.6 & 139 & 1.59 & 36 & 17.40 & 15.848222 & 8.9 \\
12 & 398.5 & 133 & 1.56 & 36 & 41.30 & 37.741438 & 8.6 \\
13 & 826.5 & 141 & 1.83 & 35 & 80.75 & 72.281379 & 10.5 \\
14 & 272.0 & 140 & 1.76 & 36 & 136.75 & 122.02401 & 10.8 \\
15 & 377.7 & 139 & 1.57 & 33 & 49.75 & 58.099922 & 16.8 \\
16 & 408.9 & 134 & 1.58 & 38 & 6.75 & 8.73098 & 29.3 \\
\hline MAPE & & & & & & & \\
\hline
\end{tabular}

The turbine air compression ratio was increased from 1.5 to 3 and the turbine outlet temperature was increased from 32 to $40^{\circ} \mathrm{F}$. The results are shown in Figs. 11 and 12. 


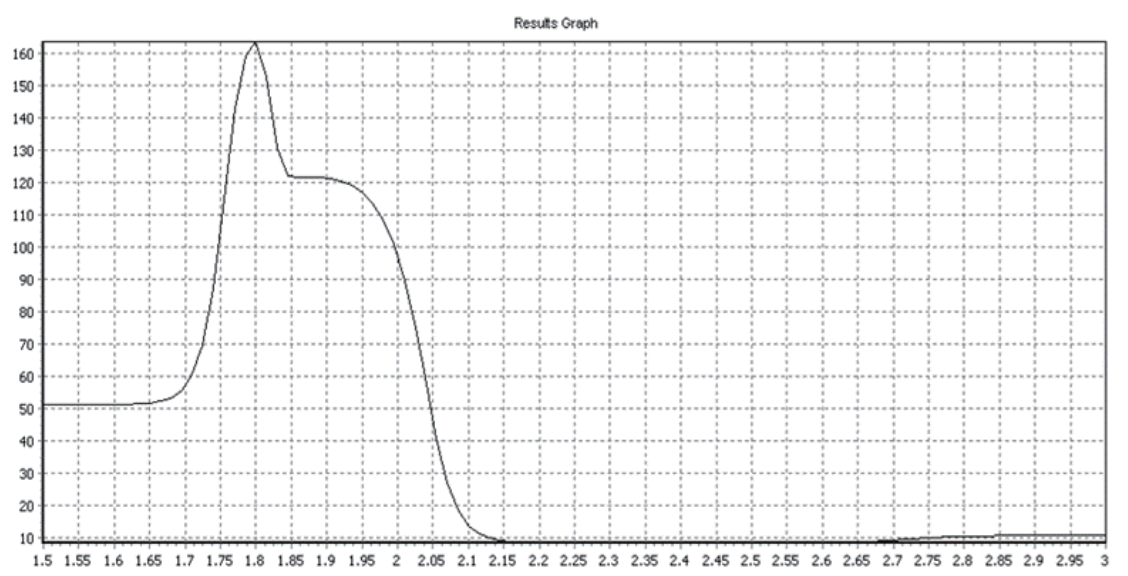

Fig. 11. Comparison between predicted and target values of turbine air compression ratio.

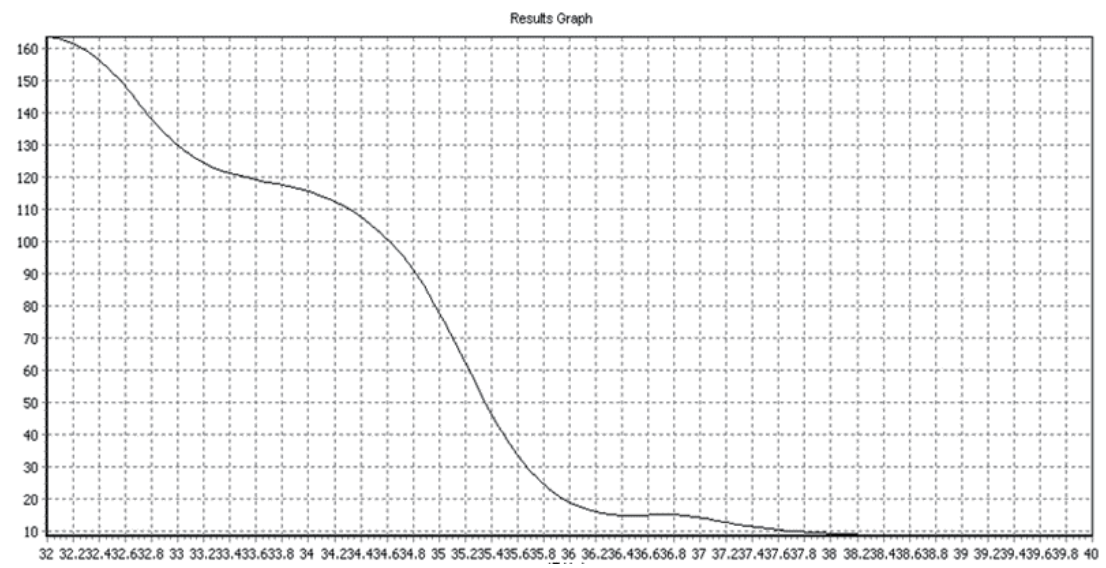

Fig. 12. Comparison between predicted and target values of turbine outlet temperature.

On the basis of the above model, the following results were obtained.

(1) Difference in sensitivity of parameters

The model sensitivity for the turbine air compression ratio was better than that for the turbine outlet temperature, which indicated that the turbine air compression ratio had a greater impact on the service life of the cooling turbine.

(2) Turbine air compression ratio

By comparing the predicted and target values of the turbine air compression ratio (Fig. 11), we found that the target value rapidly increased above a turbo air compression ratio of about 1.67, reaching its maximum value at a ratio of 1.8 .

(3) Turbine outlet temperature

The comparison of the predicted and target values of the turbine outlet temperature showed that when the working hours with the installed turbine were close to the hours after fault detection, the turbine outlet temperature increased. 


\section{Conclusion}

In this study, we used a BPNN as a predictive tool. To choose the components and parameters to establish a prediction model for training the neural network, we adopted the Delphi method. From the results of repeated questionnaire surveys, we selected six components of a jet fighter's engine: engine drain valve, primary/secondary heat exchanger, cooling turbine, regenerative heat exchanger, dehydrator, and sensor controller of electronic equipment. Among these, the cooling turbine of the engine was chosen as the most important component to train the neural network. Then, four key parameters were selected from the questionnaire results: air compression ratio in the turbine, turbine outlet temperature, flight hours (operation hours) with the installed turbine, and exhaust air pressure of the engine. We input the actual inspection data for training the neural network and performed a model verification and a comparison between the actual data and the predicted data. Repeated verification proved that the prediction model was accurate.

In accordance with the current regulations and relevant policies, ${ }^{(8)}$ a jet fighter aircraft must undergo a periodic inspection every 200 flight hours, which includes an inspection of the environmental control system. Using the predicted data from the prediction model, the following maintenance strategy is recommended.

(1) Less than 50 predicted remaining working hours to the next inspection

The cooling turbine should not be run. Malfunctioning components detected in a thorough inspection must be changed or repaired. A jet fighter aircraft with a cooling turbine can be used only for limited training.

(2) Between 50 and 200 predicted remaining working hours

The prediction model of this study had $90 \%$ accuracy. Thus, when the working hours of the cooling turbine reaches $90 \%$ of the predicted life, prediction by the model must be performed again to avoid unnecessary testing and ensure the reliability of components.

(3) More than 200 predicted remaining working hours

A jet fighter aircraft can use the cooling turbine, and the data from the sensors in the environmental monitoring system are monitored to prevent the unexpected failure of the system.

The above maintenance strategy effectively solves the problems originating from the current short cycle and high-frequency testing, enabling the working hours of components to be extended and avoiding unnecessary wear and tear. The testing cost and labor are also reduced. The maximum working hours of cooling turbines will be achieved when the turbo air compression ratio becomes 1.8. The turbine outlet temperature can also be used for the setting as it is inversely proportional to the number of working hours, which can be used as a simple reference to predict the remaining operation hours of a cooling turbine.

The prediction model in this study can be used as a reference for developing a preventive maintenance management strategy for jet fighter aircraft that balances the availability for missions and cost saving. In addition, the methodology of developing a prediction model using the Delphi method and a BPNN can be applied to the inspection of other machines to decrease the frequency of maintenance through accurate prediction. 


\section{References}

1 D. Rengasamy, H. P. Morvan, and G. P. Figueredo: Proc. 21st Int. Conf. Intelligent Transportation Systems (ITSC, 2018) 150-156. https://doi.org/10.1109/ITSC.2018.8569502

2 A. Sahay: Leveraging Information Technology for Optimal Aircraft Maintenance, Repair and Overhaul (MRO) (Elsevier, Amsterdam, 2012) Chap. 1

3 M. McFadden and D. Worrells: IJATEM 1 (2012) 4. https://doi.org/10.5703/1288284314659

4 M. Y. Hsieh: Integrating Models of the Weapons System Combination and Life Cycle Cost, Doctoral Thesis (National Defense University, 2008) https://hdl.handle.net/11296/qt8z77 (accessed April 2020).

5 N. P. Kumar, P. K. Devarajan, S. A. Vendan, and N. Shanmugam: Int. J. Adv. Manuf. Tech. 93 (2017) 385. https://doi.org/10.1007/s00170-016-9562-8

6 M. Norpah and M. Z. A. Sakinah: Appl. Math. Com. Int. 8 (2019) 67. http://dspace.unimap.edu.my/xmlui/ handle/123456789/63715

7 J. A. Rosero, J. A. Ortega, E. Aldabas, and L. Romeral: IEEE Aero. El. Syst. Mag. 22 (2007) 3. https://doi. org/10.1109/MAES.2007.340500

8 D. V. Mahindru and P. Mahendru: Global J. Res. Eng. 11 (2011). https://engineeringresearch.org/index.php/ GJRE/article/download/234/195 